\title{
Tailored ramp wave generation in gas gun experiments
}

\author{
Matthew Cotton ${ }^{1, \text { a }}$, David Chapman ${ }^{2}$, Ron Winter ${ }^{1}$, Ernie Harris ${ }^{1}$, and Daniel Eakins ${ }^{2}$ \\ ${ }^{1}$ AWE Plc, Aldermaston, Reading RG7 4PR, UK \\ ${ }^{2}$ Institute of Shock Physics, Imperial College London, Prince Consort Road, London SW7 2AZ, UK
}

\begin{abstract}
Gas guns are traditionally used as platforms to introduce a planar shock wave to a material using plate impact methods, generating states on the Hugoniot. The ability to deliver a ramp wave to a target during a gas gun experiment enables access to different regions of the equation-of-state surface, making it a valuable technique for characterising material behaviour. Previous techniques have relied on the use of multi-material impactors to generate a density gradient, which can be complex to manufacture. In this paper we describe the use of an additively manufactured steel component consisting of an array of tapered spikes which can deliver a ramp wave over $\sim 2 \mu$ s. The ability to tailor the input wave by varying the component design is discussed, an approach which makes use of the design freedom offered by additive manufacturing techniques to rapidly iterate the spike profile. Results from gas gun experiments are presented to evaluate the technique, and compared with 3D hydrodynamic simulations.
\end{abstract}

\section{Introduction}

It is well known that experiments to measure the changing profiles of ramp compression waves can provide useful data on the material through which the wave propagates (for example see [1,2]). By introducing a shockless compression to a material the temperature change is minimised, and the pressure states generated in the target are described more closely by the isentrope. Quasiisentropic loading has traditionally been achieved through the use of magnetic or laser drive systems, which operate over very short (ns) timescales. Gas gun generated plate impacts can provide longer duration loading of materials of interest, and also benefit from the relative availability and cost of the experimental platforms. However, gas guns are better suited to introducing shock waves to a target due to the discontinuous change in pressure generated by high speed impact between two plates. A feasible method of generating a ramp wave under impact conditions is through the use of a flyer whose density is low at the leading face but increases with distance from that face. Previously this has been achieved through the use of a layered impactor consisting of multiple materials of varying density [3-5], using powder metallurgical or sedimentation techniques. These have produced good results but can be complex to manufacture, with layer thickness being a significant factor in the quality of the ramp profile. In this paper we explore the ability of a single material graded density flyer produced by an additive manufacturing technique known as selective laser melting (SLM) to generate ramp waves in a target, while minimising the initial shock rise.

A stainless steel flyer was designed which consisted of an array of tapered spikes attached to a solid base plate, referred to as the Bed-of-Nails $(\mathrm{BoN})$ design. The tapered outer profile produces the continuous density

\footnotetext{
${ }^{a}$ Corresponding author: matthew. cotton@awe.co.uk
}

gradation which is required to deliver a ramp wave to the target upon impact. Using a 3D CAD model of the BoN component the Sandia National Laboratories code $\mathrm{CTH}$ was used to model the wave delivered to a series of solid targets at a range of impact velocities. Based on this computational study experiments were designed with the objective of demonstrating the bed-of-nails concept for generating ramp waves and of determining the validity of $\mathrm{CTH}$ to compute the configuration.

\section{BoN design}

The choice of SLM as the manufacturing route for the BoN components was based on the ability of the process to produce complex geometries at small scales. The process involves depositing $40 \mu \mathrm{m}$ layers of stainless steel powder onto a base plate, which is then selectively melted by application of a $200 \mathrm{~W} \mathrm{CW}$ laser. The regions to be melted are specified by a 3D model of the desired component. When one layer is complete another powder layer is deposited until the structure is complete. In this case the BoN design consists of an array of tapered spikes of length $5.5 \mathrm{~mm}$ with a $1 \mathrm{~mm}^{2}$ base, lying on a $2.5 \mathrm{~mm}$ thick base plate. A 3D model of the design is shown in Fig. 1.

For the initial design a parabolic outer profile was chosen for the spikes to deliver a linear variation in areal mass along the impact axis. The parabola which defines the outer surface can be expressed as:

$$
z=25-6 x^{2}
$$

Subsequent investigations have explored the effects of varying the profile to change the mass distribution and thus the shape of the wave delivered to the target upon impact, the results of which are described later in this paper.

Upon receipt of the completed BoN specimens, a sample was sectioned and a series of micrographs taken to 


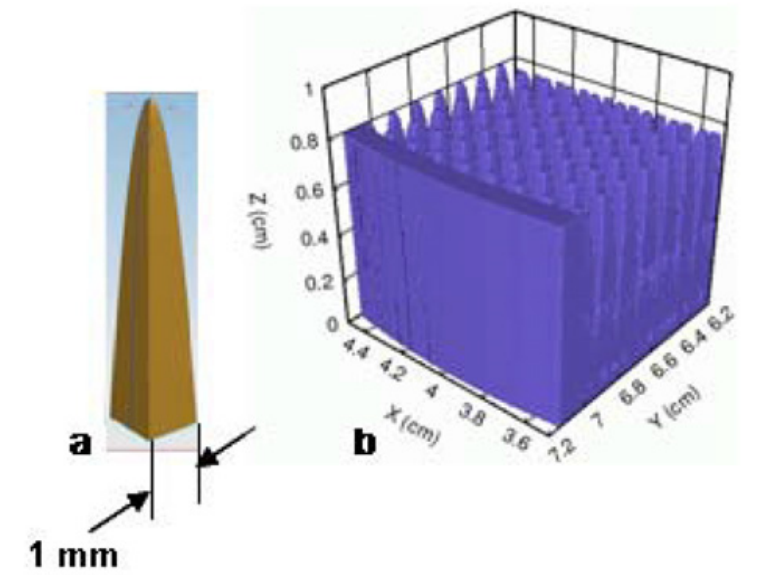

Figure 1. (a) Individual spike with parabolic outer profile. (b) a section of the BoN array with outer retaining ring.

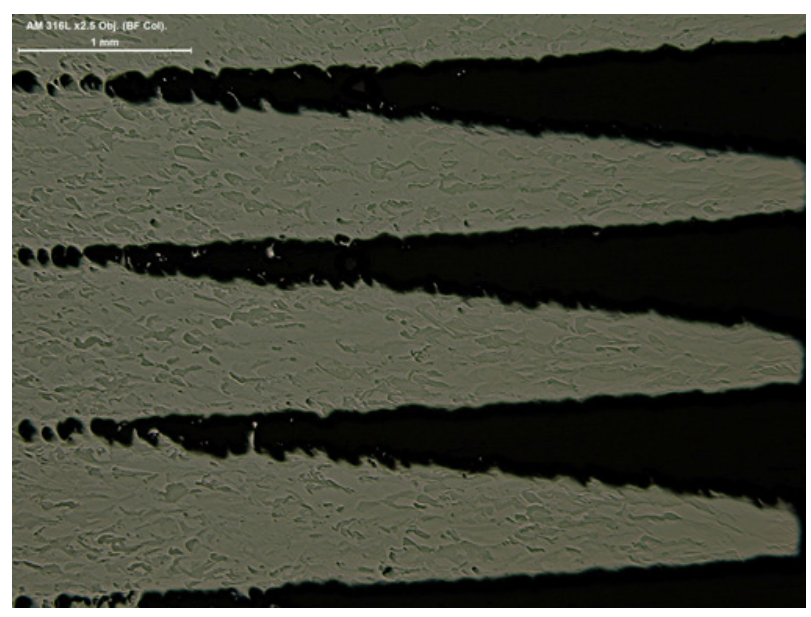

Figure 2. Cross-section of the spikes. The spikes join together above the nominal contact point due to the additional layer of powder particles.

establish how accurately the SLM process had reproduced the features in the CAD model. These images displayed some additional deposition of partially melted powder particles on the outer surface of the spikes which led to an increase in the overall mass of the BoN compared to nominal values. An example of this phenomenon is shown in Fig. 2.

Similar observations regarding surface finish have been made by several authors who have utilised SLM [68]. From these investigations it can be concluded that a number of factors including laser power, movement speed and scanning direction all contribute to characteristics of the completed part. Options exist for post-processing of the SLM part to minimise the effects of surface roughness, but in this case the BoN components were used in the asreceived condition.

\section{CTH simulations}

A series of simulations of the experimental geometry have been conducted using CTH, a 3D Eulerian hydrocode [9]. This code was selected due to its ability to import the same 3D CAD models which were used to manufacture the

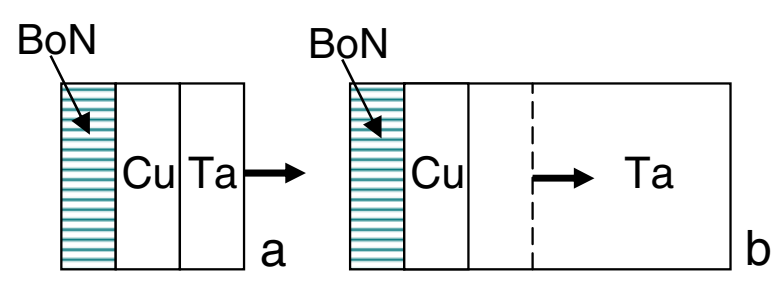

Figure 3. Calculational setup for (a) free surface and (b) in-situ wave profiles.

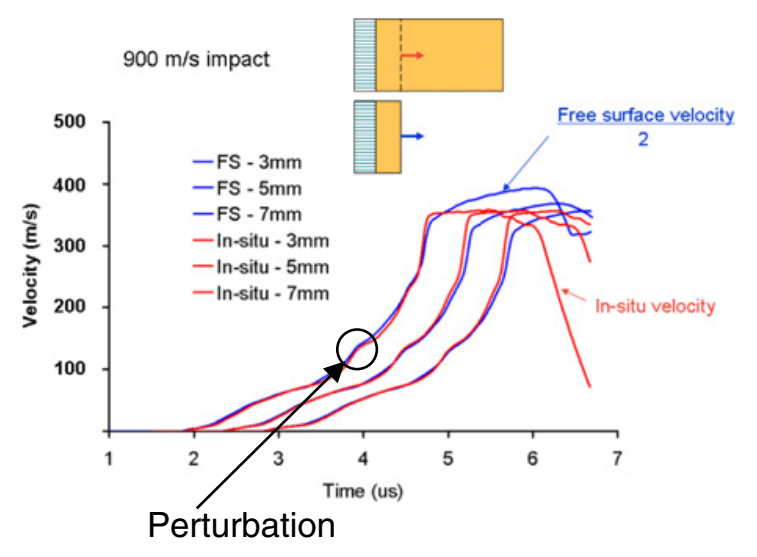

Figure 4. Calculated velocity profiles for both in-situ particle velocity and half the free surface velocity.

BoN components during the SLM process, as well as its suitability for modelling high strain rate impact processes.

In order to reduce the computational workload a reduced version of the BoN CAD model, consisting of a $3 \times 3$ array of spikes, was used for most simulations. It was established beforehand that the use of the reduced model did not introduce any significant variation in the results. For all simulations a $50 \mu \mathrm{m}$ mesh was used, and a Johnson Cook strength model was assigned to the materials. As discussed above, some discrepancies were noted between the CAD model and the manufactured components. An updated model with an increased total mass was generated by adjusting the equation of hte parabola and used to make comparisons with the experimental data.

The calculational geometry is illustrated in Fig. 3. The BoN component impacts a $6 \mathrm{~mm}$ thick copper transmitter backed by a tantalum plate of varying thickness, at an impact velocity of $900 \mathrm{~m} / \mathrm{s}$. The data was output in the form of velocity-time profiles from the rear surface of the target (a) to allow it to be compared to the experimental results. In-situ velocities (b) were also output to estimate the error introduced by using a free surface approximation (in-situ velocity, $u_{p}$, is half the free surface velocity, $u_{\mathrm{fs}}$ ) in our analysis.

A copper plate was placed between the BoN impactor and the tantalum target to smooth out any discontinuities in the input wave prior to it entering the target.

The results of these simulations are shown in Fig. 4.

The two profiles are near identical during the ramp phase, which gives confidence in the use of free surface velocity data in our subsequent analysis. An evaluation of the velocity discontinuities was based on the velocity plots from the $3 \mathrm{~mm}$ target, shown in Fig. 5. 


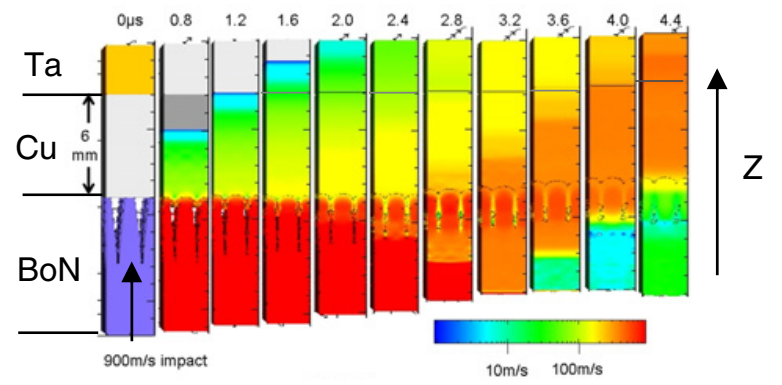

Figure 5. Velocity plots for a $900 \mathrm{~m} / \mathrm{s}$ impact on to a copper/tantalum target.

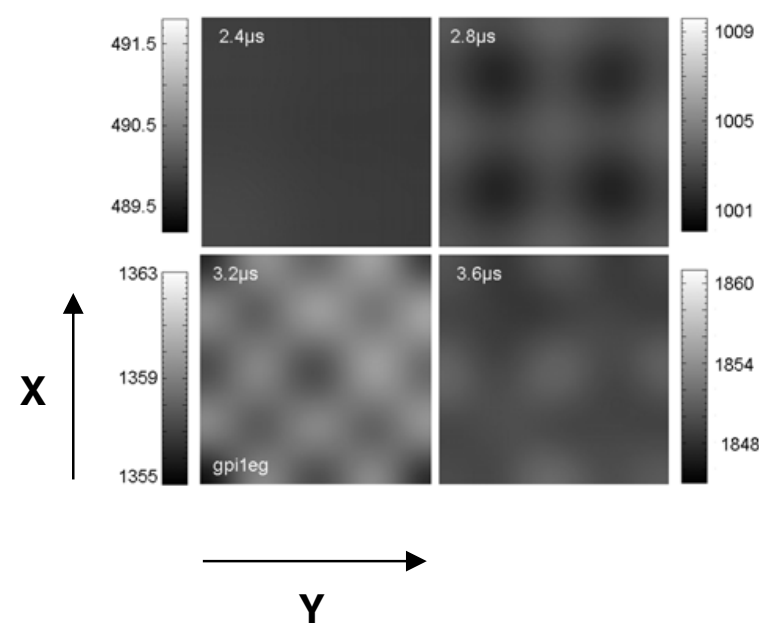

Figure 6. Velocity distribution across a $2 \mathrm{~mm}^{2}$ region in the $\mathrm{x}-\mathrm{y}$ plane at the rear of a $3 \mathrm{~mm}$ thick tantalum disc.

The results demonstrate that velocity perturbations along the z-axis are rapidly smoothed out as the wave propagates into the copper buffer plate. A small discontinuity appears to be introduced to the copper around $2.8 \mu$ s after impact, which may be responsible for the small discontinuity visible in the trace approximately half way along the ramp.

The effect of perturbations in the $x-y$ plane (where $\mathrm{z}$ is the impact axis) were also assessed to determine if the non-uniform pressure distribution at the impact face propagated though the target to the proposed diagnostic recording location. Figure 6 shows the calculated velocity distribution at the rear surface of the tantalum disc as the wave is reflecting from the free surface.

These results indicate that the in-plane velocity varies by only $\sim 0.5 \%$ from the mean velocity, giving confidence that any perturbations introduced by the spikes upon impact will have been damped as they pass through the target.

The results from these simulations provided confidence in the ability of the design to deliver a ramp wave, and experimental trials were conducted using this configuration.

\section{Experimental data}

The initial trial series consisted of two experiments conducted at nominal impact velocities of 900 and
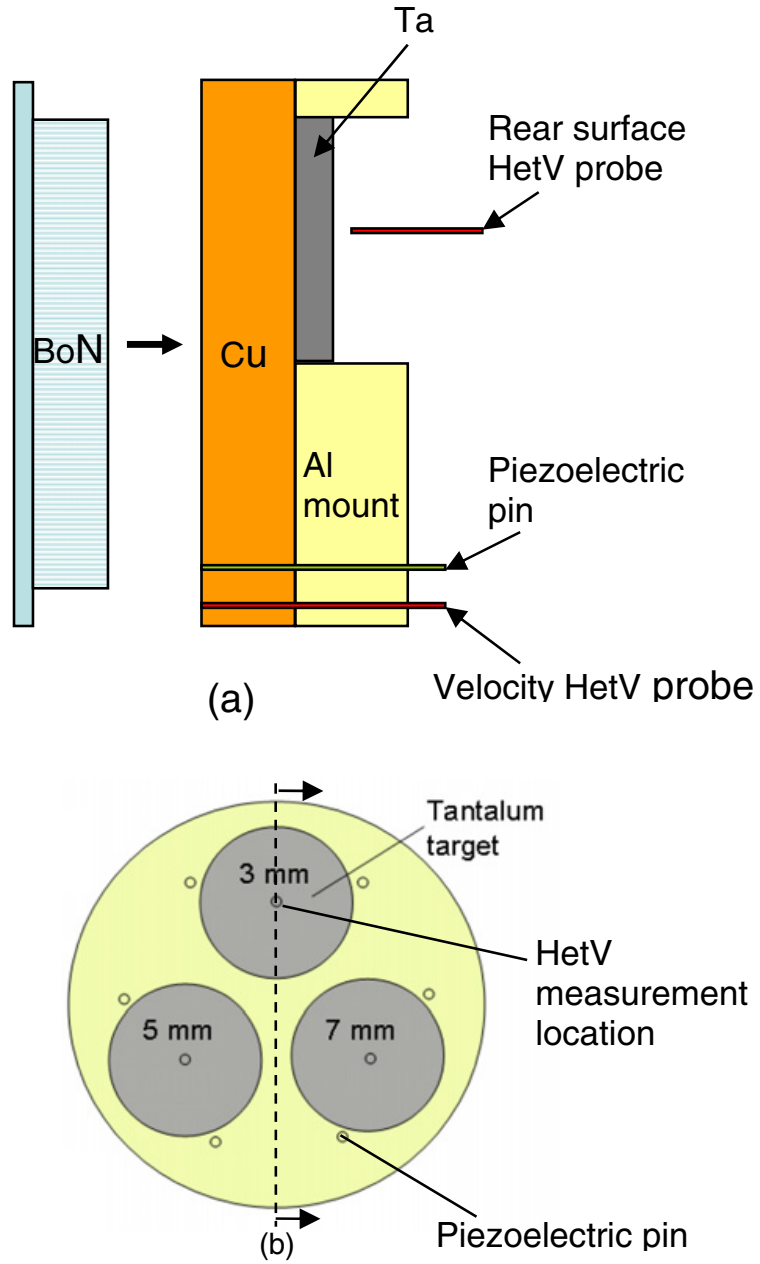

Figure 7. (a) Schematic section and (b) rear surface views of the experimental assembly.

$1100 \mathrm{~m} / \mathrm{s}$. The target consisted of a copper transmitter plate with three tantalum discs of varying thickness mounted on the rear surface. The principal diagnostic used was heterodyne velocimetry (HetV) which recorded velocitytime profiles from the rear surface of the tantalum discs. Piezoelectric pins were also used to provide time of arrival information at the copper/tantalum interface. The principal features of the experimental assembly are illustrated in Fig. 7. The BoN impactor is shown mounted on a polycarbonate sabot in Fig. 8.

The two experiments were fired at impact velocities of 900 and $1100 \mathrm{~m} / \mathrm{s}$. The higher velocity impact experiment supplied poor timing data, so the subsequent analysis shown here is confined to the lower velocity shot.

The measured velocity profiles from the three tantalum targets from the lower velocity shot are displayed in Fig. 9, alongside the $\mathrm{CTH}$ predictions.

The experimental data confirms that the BoN impactor has delivered a ramp wave to the target over a period of $\sim 2.5 \mu \mathrm{s}$. An initial elastic wave can also be distinguished up to $\sim 50 \mathrm{~m} / \mathrm{s}$, representing the isentropic elastic limit. The ramp wave transitions to a shock near to the peak velocity, apparent as a notable steepening of the gradient. For the purposes of ramp wave analysis, this transition marks the end point of the experiment. The arrival of 


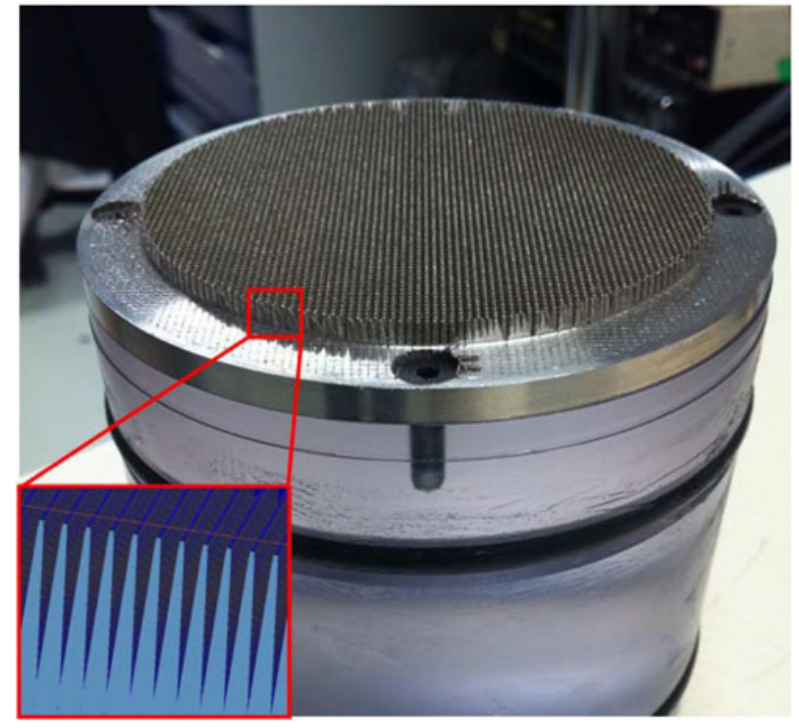

Figure 8. BoN impactor mounted on a polycarbonate sabot note machined edge to provide a reflective surface for velocity measurement using HetV.

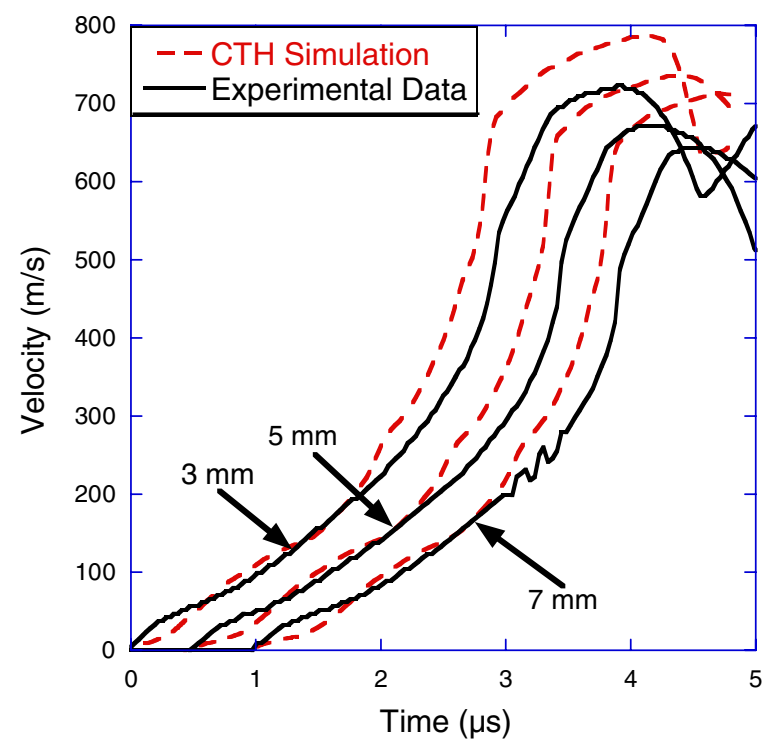

Figure 9. Experimental rear surface velocity data for each Ta target, and CTH simulations for comparison. $0 \mu$ s corresponds to wave arrival at the rear of the $3 \mathrm{~mm}$ Ta target.

a release wave from the rear of the flyer causes a further change in gradient corresponding to a reduction in acceleration. Peak velocity is observed to decrease as target thickness increases, an effect caused by the faster moving release wave overtaking the compression wave as it propagates further into the target. Similar features were observed in ramp loading data gathered using magnetic drive techniques by Asay [10].

The simulations agree with the experimental data reasonably well over the course of the ramp, providing increased confidence in our predictive capability using $\mathrm{CTH}$. The main discrepancy is an increase in velocity at $\sim 2 \mu$ s after shock arrival at the rear surface which does not occur in the experimental data. This leads to a vertical

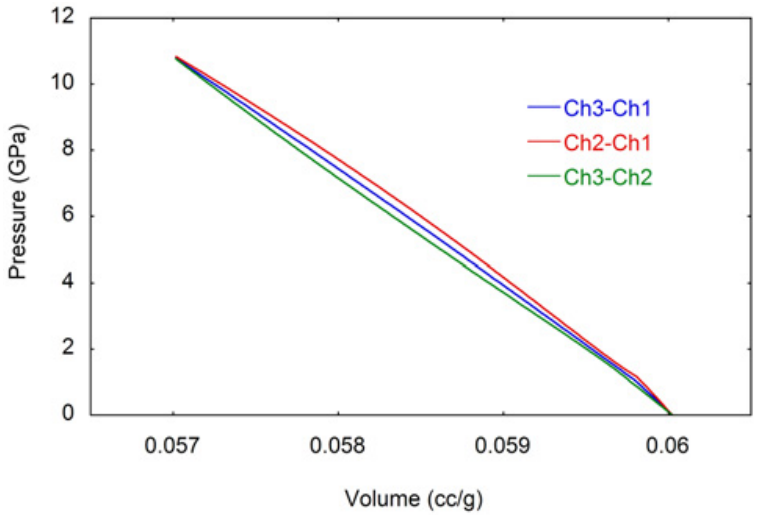

Figure 10. P-V plots of the data from the three pairs of HetV channels.

offset between the simulation and experiment for the remainder of the ramp. The time of arrival suggests that the velocity increase originates from a wave reflection at the BoN/copper interface caused by the density discontinuity. The fact that this feature does not appear in reality may indicate that the density change at the BoN/copper interface smoothes out post impact in a manner which is not captured in $\mathrm{CTH}$.

Using the free surface approximation, discussed above, the HetV data can be analysed to extract stress-volume points. For the region of ramp wave compression these data points should lie on the material isentrope. The following equations are used [11]:

$$
\begin{gathered}
\mathrm{C}_{L}=\left(z_{2}-z_{1}\right) /\left(t_{2}\left(u_{p}\right)-t_{1}\left(u_{p}\right)\right) \\
\mathrm{d} P=\rho_{0} \mathrm{C}_{L} \mathrm{~d} u_{p} \\
\mathrm{~d} V=-V_{0}\left(\mathrm{~d} u_{p} / \mathrm{C}_{L}\right) .
\end{gathered}
$$

Where:

$\mathrm{C}_{L}$ - Lagrangian sound speed

$\mathrm{z}-$ target thickness

$\mathrm{u}_{\mathrm{p}}-$ Particle velocity

$\mathrm{t}\left(\mathrm{u}_{\mathrm{p}}\right)$ - time at which velocity $\mathrm{u}_{\mathrm{p}}$ is reached

$\mathrm{P}$ - Pressure

V - Volume

Subscripts 1 and 2 refer to the two Ta targets which are being used in the analysis.

The isentrope can be calculated using velocity profiles at two different points on the $\mathrm{z}$ axis, therefore the three targets fielded in this experiment provide three routes to accomplish this. By comparing the isentropes produced by these routes the consistency of the data can be evaluated. This data is shown in Fig. 10.

Encouragingly the data does appear to be fairly consistent, and the small deviations that do occur are likely to result from timing errors which contribute to the majority of the $\sim 2-3 \%$ uncertainty in $\mathrm{C}_{L}$.

\section{Revised design}

Following the initial series of experiments the design of the BoN was adjusted with the aim of improving the linearity 


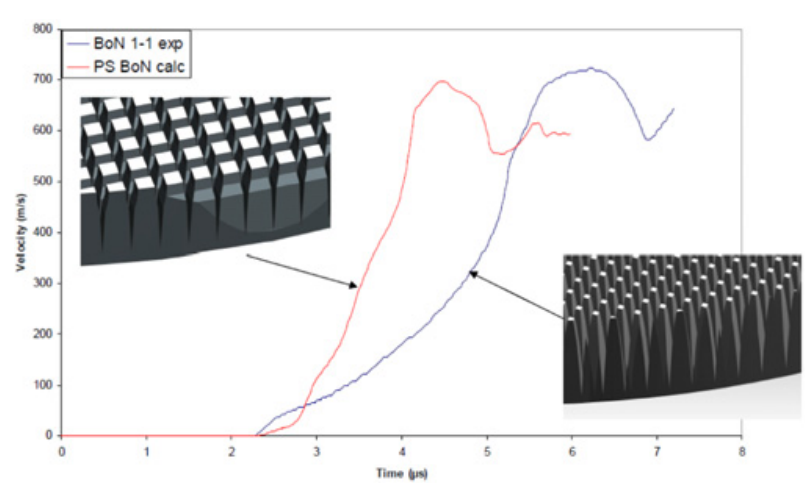

Figure 11. Comparison of BoN (right) and PSBoN (left) wave profiles.

of the ramp wave supplied by the technique. The objective was to introduce a wave which delivers a shorter ramp with a more linear increase in velocity. This adjustment is intended to reduce the effects of wave reflections from the rear of the flyer which can complicate the analysis. To achieve this the original BoN design went through a number of iterations which were simulated in CTH to assess the changes in the wave profile. The changes in mass distribution along the nail led to a more complex stepped profile being generated, referred to as the Parabolic Step Bed-of-Nails (PSBoN). A number of modifications were also made to the target design with the aim of minimising the errors in timing. Principal among these was the introduction of additional HetV probes to provide timing information at the copper/tantalum interface. A comparison of the original BoN wave profile with the predicted PSBoN profile is shown in Fig. 11.

This method enables the desired input wave to be specified based on the experimental requirements. As the technique matures it is expected to deliver a capability to generate more complex wave profiles based on a combination of shock, ramp and release stages. These options will be investigated further following the conclusion of the PSBoN experiments.

\section{Conclusions}

A novel technique for delivering ramp waves to a target in a gas gun plate impact experiment has been demonstrated. The BoN design delivered a smoothly ramped wave to the tantalum targets with minimal initial shock upon impact, and data quality is comparable to that produced by other gas gun graded density impactors. Subsequent analysis of free surface wave profiles has yielded data on the tantalum isentrope. A series of simulations of the experiments has also demonstrated that the $\mathrm{CTH}$ hydrocode can reproduce the principal features of the wave profiles, and can therefore be applied as a predictive tool in the design phase.

The ramp wave generation relies on the use of an additively manufactured component which utilises the design freedom offered by this processing route to make the BoN impactors. This technique also offers the opportunity to tailor the input wave profile by altering the design of the impactor, a method which has been demonstrated through the development of the PSBoN variant. Future work will explore the possibility of creating more complex wave profiles using the same design methodology.

The authors would like to thanks Neil Bond (AWE) for supplying the optical microscopy, Nick Gibbs (AWE) for assisting with the design of the BoN component, and Steve Johnson (Imperial College London) for experimental support. AWE and Imperial College London are acknowledged for their continued support of the Institute of Shock Physics.

\section{References}

[1] J-P. Davis, J. Appl. Phys. 99, 103512 (2006).

[2] J.R. Asay, T.J. Vogler, T. Ao, J.L. Ding, J. Appl. Phys. 109, 073507 (2011).

[3] J.H. Nguyen, D. Orlikowski, F.H. Streitz, J.A. Moriarty, N.C. Holmes, J. Appl. Phys. 100, 023508 (2006).

[4] H. Xiong, L. Zhang, L. Chen, R. Yuan, T. Hirai, Metall. Mater. Trans. A 31, 2369 (2000).

[5] L.P. Martin, J.R. Patterson, D. Orlikowski, J.H. Nguyen, J. Appl. Phys. 102, 023507 (2007).

[6] G. Strano, L. Hao, R.M. Everson, K.E. Evans, J. Mat. Proc. Tech. 213, 589 (2013).

[7] M. Smith, W.J. Cantwell, Z. Guan, S. Tsopanos, M.D. Theobald, G.N. Nurick, G.S. Langdon, J. Sand. Struct. Mat. 13, 479 (2010).

[8] S. Van Bael, G. Kerckhofs, M. Moesen, G. Pyka, J. Schrooten, J.P. Kruth, Mat. Sci. Eng. A 528, 7423 (2011).

[9] J.M. McGlaum, S.L. Thompson, M.G. Elrick, Int. J. Imp. Eng. 10, 351 (1990).

[10] J.R. Asay, T. Ao, T.J. Vogler, J-P. Davis, G.T. Gray, J. Appl. Phys. 106, 073515 (2009).

[11] S.D. Rothman J.R. Maw J. De Physique IV 134, 745 (2006). 